\title{
L'ICHTHYOFAUNE MESSINIENNE DU BASSIN DE SORBAS (ALMERÍA, ESPAGNE) ET SES RAPPORTS AVEC L'ENVIRONNEMENT SÉDIMENTAIRE
}

\author{
Jean GAUDANT \\ 17 rue du Docteur Magnan 75013 Paris (Département Histoire de la Terre du \\ Muséum national d'Histoire naturelle: USM 203 et UMR 5143 du C.N.R.S.)
}

Gaudant, J. 2008. L'ichthyofaune messinienne du bassin de Sorbas (Almería, Espagne) et ses rapports avec l'environnement sédimentaire. [The Messinian fish fauna of the Sorbas basin (Almería, Spain) and its relation with the sedimentary environment.] Revista Española de Paleontología, 23 (2), 211-223. ISSN 0213-6937

\begin{abstract}
Fish skeletons were collected at three different levels in the Messinian of the Sorbas basin (Andalusia, South Spain). The richest fossiliferous level is a diatomitic stratum which occurs a few metres below the bottom of the evaporitic sequence. It has mainly yielded many juvenile Myctophids: Myctophum licatae and less abundant Maurolicus muelleri This indicates the occurrence of a rather deep open sea in the vicinity. Additionally, some skeletal remains of Sardina ? crassa were found in a clayey intercalation between two massive beds of gypsum. The occurrence of this marine species is indicative of the persistence of the sea during the evaporitic episode. Finally, as shown by the occurrence of Aphanius crassicaudus in the postevaporitic Messinian, a lagoonal environment existed in this basin, as a result of a relative lowering of the sea level. Additionally, another fossiliferous locality yielding the same species is reported near Gafares, in the postevaporitic Messinian of the northern part of the Níjar-Carboneras basin.
\end{abstract}

Key-words: Fishes, Teleosts, Upper Miocene, Palaeoecology, Palaeobathymetry, Spain.

\section{RÉSUMÉ}

Des squelettes de poissons ont été récoltés à trois niveaux dans le Messinien du bassin de Sorbas (Andalousie, Sud de l'Espagne). L'horizon le plus fossilifère est un lit diatomitique situé à quelques mètres au-dessous de la base de la séquence évaporitique. Il a principalement livré de nombreux Myctophidae juvéniles: Myctophum licatae et des Maurolicus muelleri moins abondants, ce qui indique la présence d'une mer ouverte relativement profonde aux environs. D'autre part, quelques squelettes plus ou moins complets de Sardina ? crassa ont été découverts dans une intercalation argileuse entre deux bancs de gypse massif. La présence de cette espèce marine témoigne de la persistance de la mer durant l'épisode évaporitique. Enfin, comme le montre la présence d'Aphanius crassicaudus dans le Messinien postévaporitique, un milieu lagunaire s'est établi dans le bassin, suite à un abaissement relatif du niveau marin. Un autre gisement de cette même espèce est en outre signalé près de Gafares, dans le Messinien postévaporitique du nord du bassin de Níjar-Carboneras.

Mots-clés: Poissons, Téléostéens, Miocène supérieur, Paléoécologie, Paléobathymétrie, Espagne.

\section{RESUMEN}

Se han obtenido esqueletos de peces de tres niveles diferentes en el Messiniense de la Cuenca de Sorbas (Andalucia, Sur de España). El nivel fosilífero más rico corresponde a un estrato diatomítico que está situado a unos pocos metros por debajo de la base de la secuencia evaporítica. En este estrato se han recuperado numerosos juveniles de la familia Myctophidae: Myctophum licatae y, en menor medida, Maurolicus muelleri, lo que indica la presencia en el paleoambiente de un mar abierto relativamente profundo. Además, se han encontrado varios restos esqueléticos de Sardina ? crassa en una intercalación arcillosa entre los niveles masivos de yeso. La presencia de esta especie marina indica la persistencia del mar durante el episodio evaporítico. Finalmente, como indica la presencia de Aphanius crassicaudus en el Messiniense postevaporítico, se estableció un ambiente lagu- 
nar en la cuenca como resultado de una bajada relativa del nivel del mar. Además, se presenta otro yacimiento con presencia de la misma especie cerca de Gafares, en el Messiniense postevaporítico de la parte norte de la cuenca de Níjar-Carboneras.

Palabras clave: Peces, Teleósteos, Messiniense, Mioceno superior, Paleoecología, Paleobatimetría, España.

\section{INTRODUCTION}

Le Messinien du bassin de Sorbas (Province d'Almería, Andalousie) (Figs 1,2) a livré des poissons fossiles à trois niveaux successifs (Fig. 3). L'horizon le plus fossilifère se situe à environ 8 mètres au-dessous de la base du gypse, dans une passée diatomitique intercalée sous le sommet de la "Formation Abad", telle qu'elle est définie par Sierro et al. (2001). Les meilleures conditions d'affleurement sont réunies au sommet de la coupe de Molinos de Aguas. Toutefois, quelques squelettes de poissons ont également été recueillis à une vingtaine de kilomètres plus à l'ouest, à quelques mètres au-dessous des gypses de Los Yesos.

Le second niveau est constitué par une intercalation argileuse qui sépare les deux bancs principaux de gypse exploités dans la grande carrière de gypse située au-dessus de Molinos de Aguas ("Formation Yesares"). Nous y avons reconnu la présence de l'espèce Sardina ? crassa (Sauvage, 1873), comme précédemment mentionné par Néraudeau et al. (2002).

Enfin, l'empreinte d'un squelette d'Aphanius crassicaudus (Agassiz, 1839) a été récoltée par M. Gilles Conesa dans le Messinien postévaporitique ("Formation Sorbas"), à proximité d'un réservoir d'eau situé en bordure de la route de Sorbas, à environ un kilomètre au nord-ouest de la carrière de gypse de Molinos de Aguas.

On dispose ainsi d'informations sur les poissons qui peuplèrent le bassin de Sorbas pendant trois épisodes du Messinien.

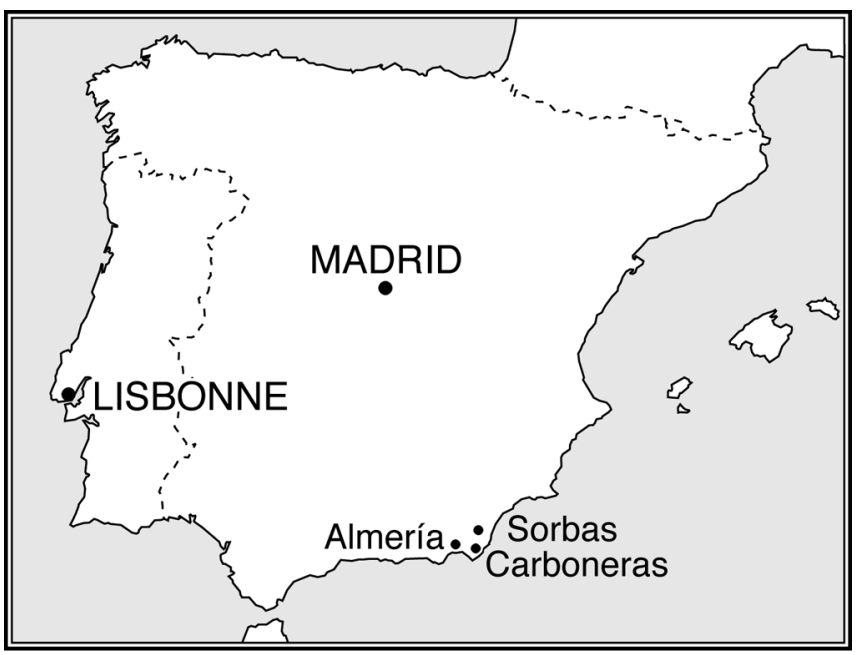

Figure 1. Carte de localisation de Sorbas et de Carboneras. Location map of Sorbas and Carboneras.

\section{L'ICHTHYOFAUNE DU MESSINIEN PRÉÉVAPORITIQUE DE MOLINOS DE AGUAS}

Le matériel décrit ci-dessous a été récolté dans un horizon diatomitique d'environ $20 \mathrm{~cm}$ ("Formation Abad", Fig. 3) d'épaisseur qui surmonte un banc de marne grise épais de $60 \mathrm{~cm}$ renfermant une malacofaune assez peu diversifiée. Ott d'Estevou (1980) fut le premier à y repérer la présence de poissons fossiles en dépit des mauvaises conditions d'affleurement dues à la présence d'une végétation dense. Ultérieurement, l'élargissement de la route provinciale AL 140 qui unit Sorbas à Carboneras, en entraînant la disparition de cette végétation, rendit plus favorables les conditions d'étude et permit la réalisation d'un échantillonnage plus substantiel dans le talus de la route, à quelques mètres au-dessous du mur de soutènement de la première courbe qui marque le début de la descente vers le Río de Aguas.

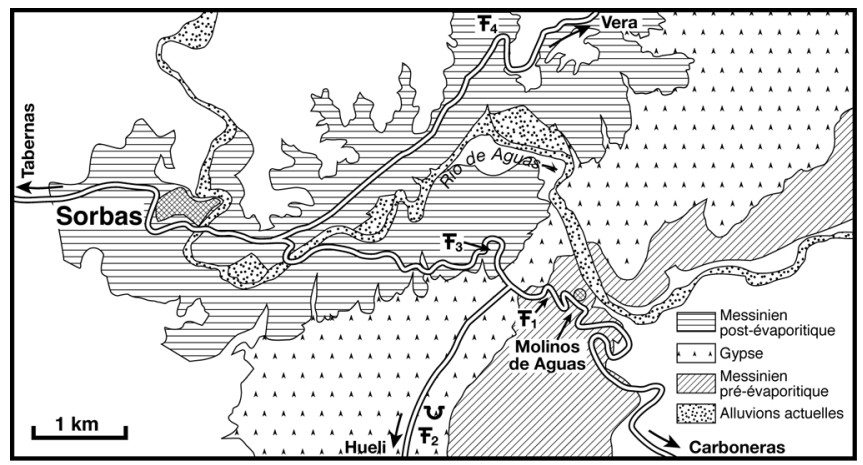

Figure 2. Carte géologique simplifiée des environs de Sorbas montrant la localisation des gisements étudiés. F 1: Messinien préévaporitique ("Formation Abad") de Molinos de Aguas; F 2: carrière de gypse de Molinos de Aguas ("Formation Yesares"); F 3: origine du squelette d'Aphanius crassicaudus (Agassiz, 1839) représenté Fig. 8; F 4: Messinien post-évaporitique ("Formation Sorbas") de Las Lomas de Sorbas (Gaudant \& Ott d'Estevou, 1985).

Simplified geological map of the surroundings of Sorbas, showing the location of the studied localities. F 1: preevaporitic Messinian ("Abad Formation”) of Molinos de Aguas; F 2: gypsum quarry ("Yesares Formation") of Molinos de Aguas; F 3: origin of the skeleton of Aphanius crassicaudus (Agassiz, 1839) figured in Fig. 8; F 4: postevaporitic Messinian ("Sorbas Formation") of Las Lomas de Sorbas (Gaudant \& Ott d'Estevou, 1985). 
On notera en outre que quelques squelettes plus ou moins fragmentaires de poissons ont été recueillis à une vingtaine de kilomètres plus à l'ouest, dans les diatomites sous-jacentes aux gypses de Los Yesos, sur le territoire de la commune de Tabernas. Nous y avons reconnu les espèces Maurolicus muelleri (Gmelin, 1789) et Myctophum (s. 1.) licatae (Sauvage, 1870). En outre, un petit Gadidae possédant trois nageoires dorsales et deux anales paraît avoir appartenu au genre Gadiculus Guichenot, 1850. Enfin un fragment de tronc en mauvais état d'un poisson dont la longueur standard devait excéder $150 \mathrm{~mm}$ témoigne de la présence d'un Clupeidae indéterminé.

L'étude de l'ichthyofaune de la diatomite de Molinos de Aguas a montré qu'elle se compose d'au moins six taxons.

\section{Matériel étudié}

Le matériel étudié dans le présent article est conservé à $\mathrm{Pa}$ ris, dans les collections paléontologiques du Muséum national d'Histoire naturelle, sous les numéros de catalogue suivants:

- MNHNP-SOR 1 à 43: Aphanius crassicaudus (Agassiz) récoltés dans le gisement de Las Lomas de Sorbas situé dans la partie inférieure de la "Formation Sorbas" (Gaudant \& Ott d'Estevou, 1985);

- MNHNP-SOR 44 à 117: matériel provenant d'une intercalation diatomitique proche du sommet de la Formation Abad;

- MNHNP-PTE 470 à 477: Sardina ? crassa (Sauvage) : materiel récolté dans une intercalation marneuse de la "Formation Yesares" (carrière de gypse de Molinos de Aguas);

- MNHNP-PTE 478: Aphanius crassicaudus (Agassiz) découvert dans la partie inférieure de la "Formation Sorbas", entre Molinos de Aguas et Sorbas;

- MNHNP-NIJ 77 à 102: Aphanius crassicaudus (Agassiz) provenant de la Rambla de Las Colmenas, près de Gafares (de la Chapelle \& Gaudant, 1987);

- MNHNP-NIJ 103 à 142: Aphanius crassicaudus (Aggassiz) récoltés au sommet du "Manco limestone" de la Loma de Los Yesares (gisement de "Gafares Pueblo", à environ $8,5 \mathrm{~km}$ à l'ouest de Carboneras.

\section{Famille Clupeidae Cuvier, 1817 Genre Sardina Antipa, 1906}

\section{Sardina ? crassa (Sauvage, 1873) Figs 4a, 7}

Un fragment en double empreinte, dont la longueur standard atteignait environ $150 \mathrm{~mm}$ a été recueilli à la base de la diatomite de Molinos de Aguas. Il témoigne de la présence de l'espèce Sardina ? crassa dans ce gisement. En dépit de son état de conservation médiocre, on y observe un opercule subrectangulaire présentant des rides disposées en éventail sur sa région antéro-ventrale. Sa colonne vertébrale était composée d'environ 45 vertèbres. Quelques écussons indiquent la présence d'une carène ventrale. Enfin, le corps est couvert d'écailles sillonnées sur lesquelles on ne distingue pas de ponctuations dans la région centrale.

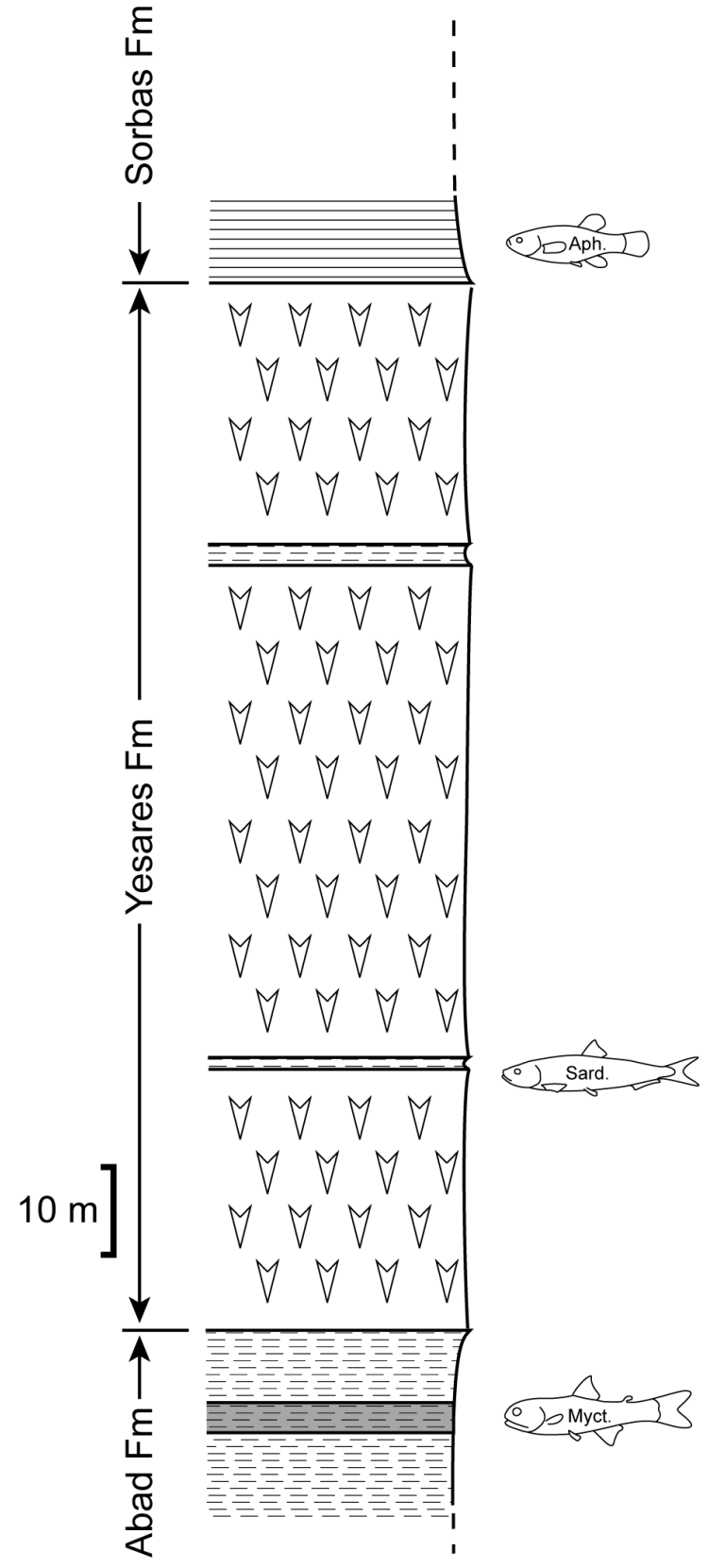

Figure 3. Coupe schématique partielle du Messinien du bassin de Sorbas montrant la position relative des trois ichthyofaunes étudiées. Fm Abad: "Formation Abad" (Messinien préévaporitique): marnes à intercalations diatomitiques; Myct.: Myctophidae. Fm. Yesares: "Formation Yesares" (Messinien évaporitique): gypse à intercalations marneuses; Sard.: Sardina?; Fm. Sorbas: "Formation Sorbas" (Messinien postévaporitique): marnes silteuses; Aph.: Aphanius.

Incomplete schematic section of the Messinian of the Sorbas basin showing the relative position of the three studied fish faunas. Fm Abad: "Abad Formation" (Preevaporitic Messinian); marls with diatomitic intercalations; Myct.: Myctophidae. Fm Yesares: "Yesares Formation" (Evaporitic Messinian); gypsum with marly intercalations; Sard.: Sardina?; Fm Sorbas: "Sorbas Formation" (Postevaporitic Messinian); marls and silts; Aph.: Aphanius. 


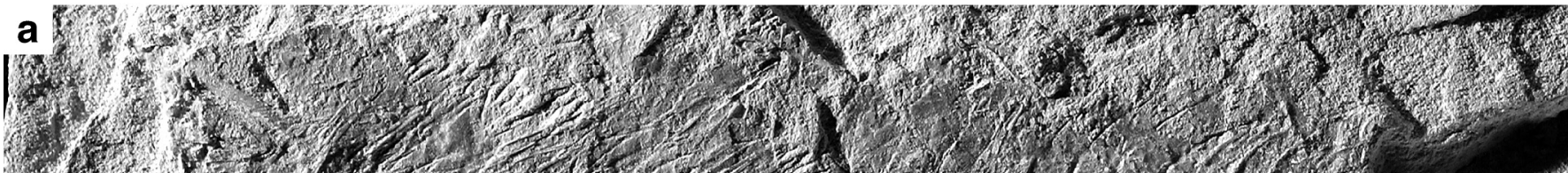

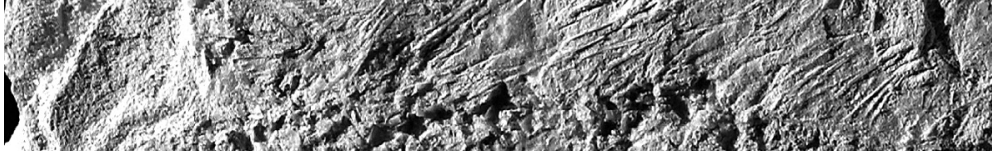
1.

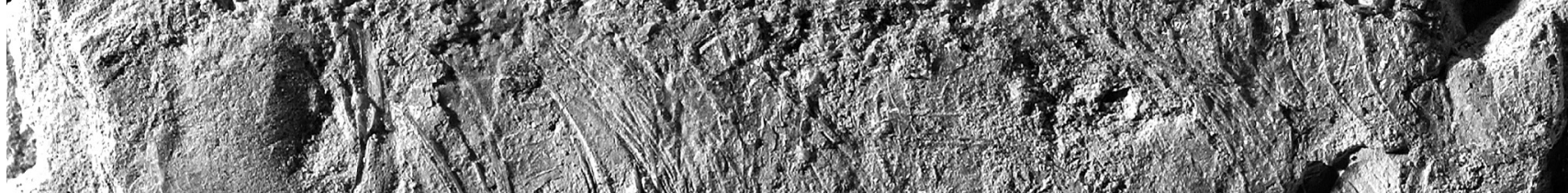
3
3

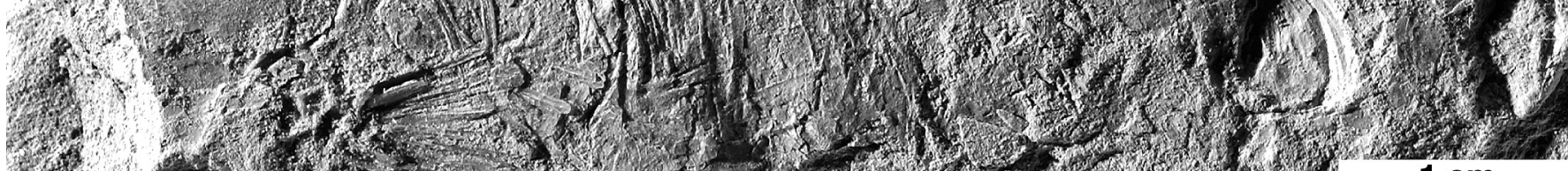

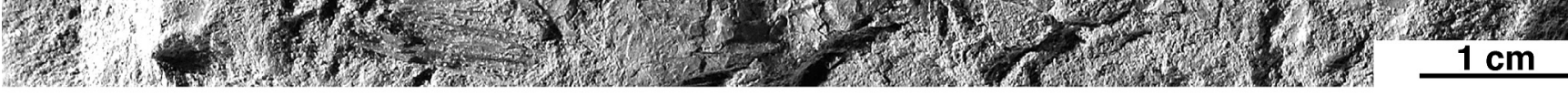
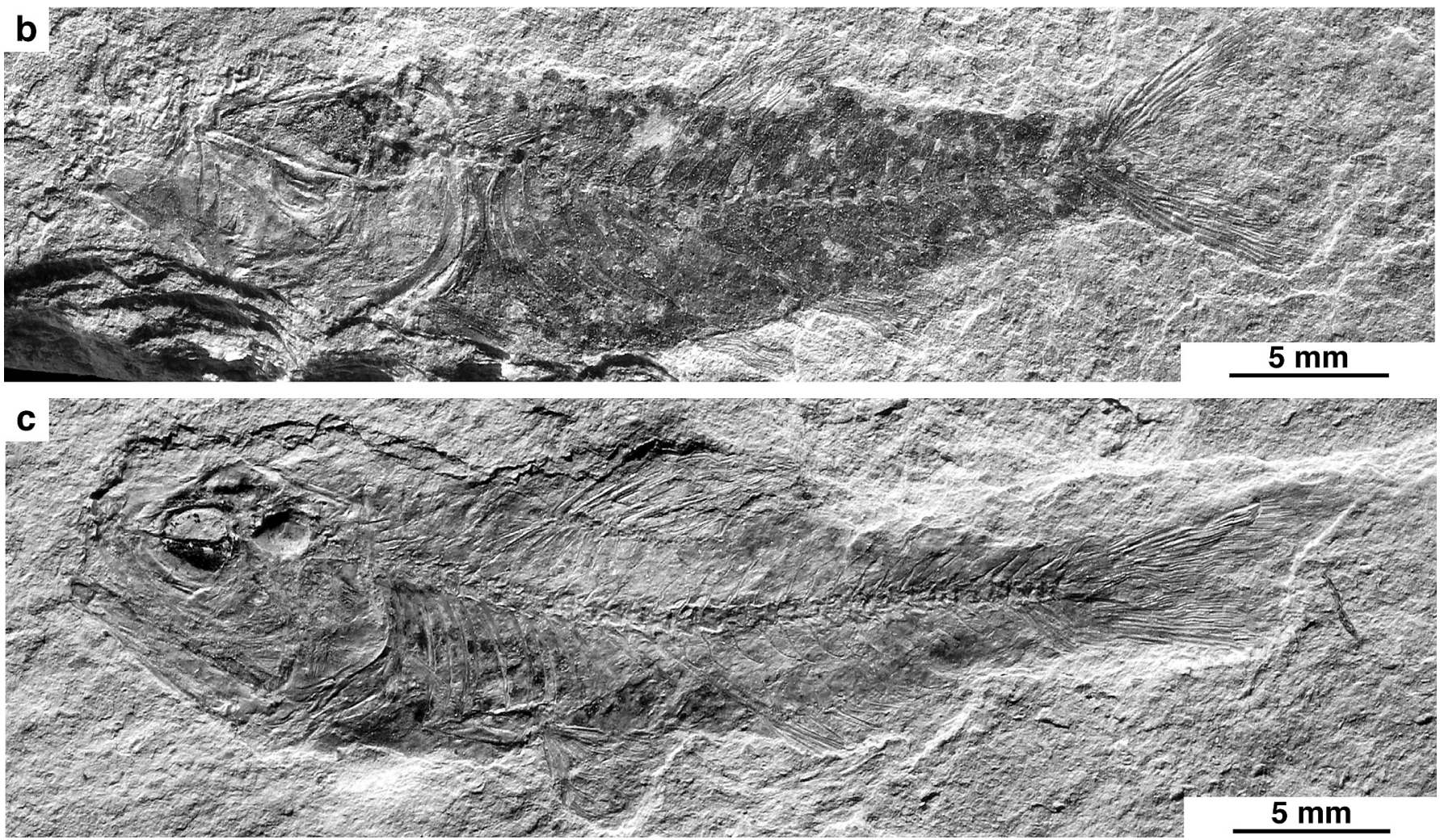

a

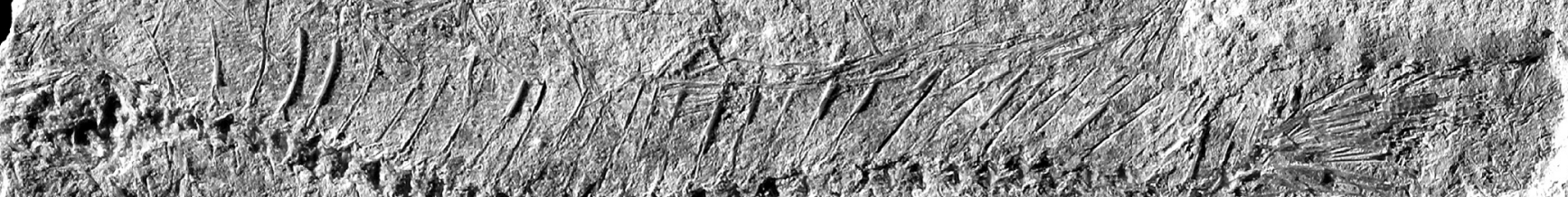
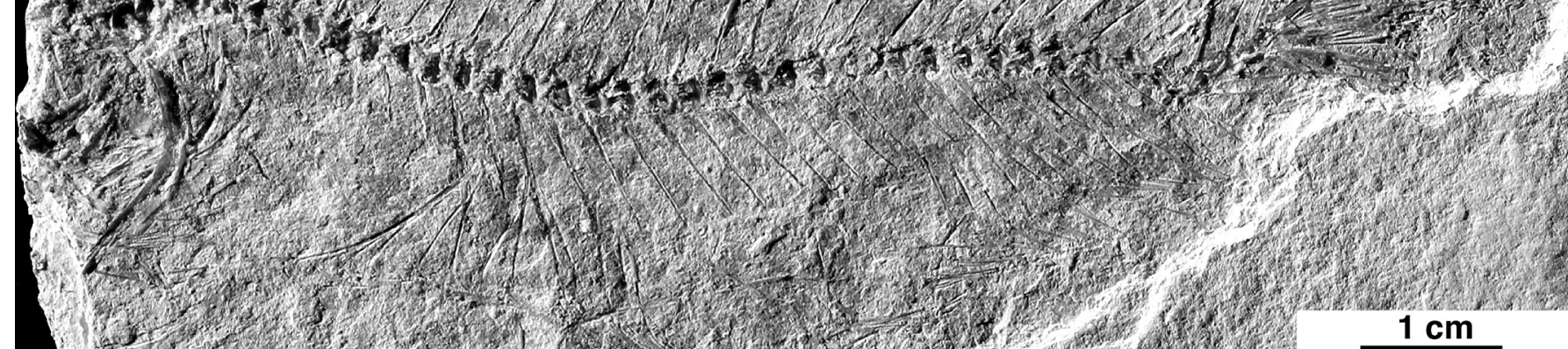
Principaux caractères diagnostiques: la forme de l'opercule et la présence de rides rayonnantes à sa surface, la composition de sa colonne vertébrale, ainsi que la possession d'écailles dépourvues de ponctuations permettent d'éliminer la possibilité qu'il s'agisse d'un représentant de l'espèce Alosa elongata Agassiz 1843 et conduisent à le rapporter à l'espèce Sardina ? crassa (Sauvage, 1873).

\section{Famille Sternoptychidae Duméril, 1806 Genre Maurolicus Cocco, 1838}

\section{Maurolicus muelleri (Gmelin, 1789)}

Fig. $4 \mathrm{~b}$

Cette espèce, qui est assez abondante dans la diatomite de Molinos de Aguas, où elle constitue 25\% du nombre d'individus récoltés, y est représentée par des individus dont la longueur standard varie de 24 à $35,5 \mathrm{~mm}$. Ce sont des poissons au corps allongé dont la hauteur maximale du corps égale environ un quart de la longueur standard.

La tête, assez massive, constitue un peu moins du tiers de la longueur du corps. Le diamètre de l'orbite égale environ un tiers de la longueur de la tête. La cavité buccale est de taille modérée car la longueur de la mandibule égale un peu moins de la moitié de la longueur de la tête. De ce fait, l'articulation de la mandibule avec le crâne prend place légèrement en avant de la verticale passant par le bord antérieur de l'orbite. Un petit prémaxillaire allongé participe au bord oral de la mâchoire supérieure.

La colonne vertébrale se compose d'environ 33 ou 34 vertèbres, dont 18 ou 19 constituent la région postabdominale. Les côtes pleurales dont le nombre n'a pas pu être déterminé, sont longues et robustes.

La nageoire dorsale prend place juste en arrière du milieu de la longueur du corps mesurée de la pointe du museau au bord postérieur des hypuraux (la distance antédorsale égale 52 à 55\% de la longueur standard). La nageoire anale débute pratiquement sur la verticale passant par la base du dernier rayon de la dorsale (la distance antéanale égale 62 à $66 \%$ de la longueur standard). Les nageoires pectorales, de taille médiocre, occupent une position basse sur le flanc. Les nageoires pelviennes, petites, sont insérées un peu en avant de la verticale passant par l'origine de la dorsale. Elles sont beaucoup plus proches de l'origine de l'anale que de la base des pectorales.

Le corps est couvert d'écailles cycloïdes.

Principaux caractères diagnostiques: la morphologie de la tête, la composition de la colonne vertébrale et la position relative des nageoires dorsale, anale et pelviennes permet- tent de s'assurer qu' on est bien en présence du genre $\mathrm{Mau}$ rolicus Cocco, 1838. Comme l'ont montré Landini \& Menesini (1980), ces poissons ne peuvent pas être distingués de l'espèce actuelle Maurolicus muelleri (Gmelin, 1789).

Famille Myctophidae Gill, 1893

Genre Myctophum Rafinesque, 1810 (s. 1.)

\section{Myctophum (s. 1.) licatae (Sauvage, 1870) Fig. 4c}

Cette espèce qui est nettement la plus abondante, avec 72,2 \% du nombre d'individus recueillis dans la diatomite de Molinos de Aguas, y est représentée par des individus dont la longueur standard varie de 9 à $34 \mathrm{~mm}$. Il s'agit donc d'alevins et d'individus juvéniles car cette espèce peut atteindre jusqu'à $115 \mathrm{~mm}$ dans le Messinien de Licata (observation inédite). Deux pics de fréquence sont observables pour des longueurs standard comprises respectivement entre 10 et $15 \mathrm{~mm}$ et entre 25 et $34 \mathrm{~mm}$. C'est une espèce au corps élancé dont la hauteur maximale du corps est comprise environ cinq fois dans la longueur standard.

La tête est grande puisqu'elle constitue approximativement le tiers de la longueur du corps. Elle est caractérisée par la grande taille de sa cavité buccale, la longueur de la mandibule atteignant environ $80 \%$ de la longueur de la tête, si bien que l'articulation de la mandibule avec le crâne prend place nettement en arrière de la verticale passant par le bord postérieur de l'orbite.

La colonne vertébrale est composée de 33 à 35 vertèbres, dont environ 18 postabdominales. La nageoire caudale est profondément fourchue.

La nageoire dorsale prend place sensiblement en avant du milieu de la longueur du corps mesurée de la pointe du museau au bord postérieur des hypuraux. La distance antédorsale égale en effet 42 à $46 \%$ de la longueur standard. Elle se compose d'une dizaine de rayons dont la longueur du plus grand égale approximativement la hauteur du corps.

La nageoire anale occupe une position reculée puisqu'elle prend place sensiblement en arrière de la verticale passant par la base du dernier rayon de la dorsale. La distance antéanale égale 65 à $68 \%$ de la longueur standard. On y dénombre une douzaine de rayons.

Les nageoires pectorales, de taille modérée, sont insérées en position basse sur le flanc. Les nageoires pelviennes sont situées un peu plus près de la base des pectorales que de l'origine de l'anale. Leur longueur égale approximativement les $2 / 3$ de la distance pelvo-anale.

Le corps est couvert d'écailles cycloïdes très fines dont la surface est ornée de circuli concentriques et dont le champ an-

Figure 4. Principaux représentants de l'ichthyofaune du Messinien préévaporitique ("Formation Abad") de Molinos de Aguas, près de Sorbas. Ce matériel est conservé à Paris, dans les collections paléontologiques du Muséum national d'Histoire naturelle [Clichés D. Serrette]. a, Sardina ? crassa (Sauvage, 1873); spécimen MNHNP-SOR 117. b, Maurolicus muelleri (Gmelin, 1789); spécimen MNHNP-SOR 50. c, Myctophum (s.1.) licatae (Sauvage, 1870); spécimen MNHNP-SOR 44. d, Arnoglossus cf. sauvagei d'Erasmo, 1930; spécimen MNHNP-SOR 114.

Main representatives of the fish fauna from the preevaporitic Messinian ("Abad Formation") of Molinos de Aguas, near Sorbas. Material kept in Paris, in the palaeontological collections of the National Museum of Natural History [Clichés D. Serrette]. a, Sardina ? crassa (Sauvage, 1873); specimen MNHNP-SOR 117. b, Maurolicus muelleri (Gmelin, 1789); specimen MNHNP-SOR 50. c, Myctophum (s. l.) licatae (Sauvage, 1870); specimen MNHNP-SOR 44. d, Arnoglossus $c f$. sauvagei d'Erasmo, 1930; specimen MNHNP-SOR 114. 
térieur paraît festonné en raison de l'existence de plusieurs radii disposés en éventail.

Principaux caractères diagnostiques: la longueur remarquable de la cavité buccale, liée à la position très reculée de l'articulation de la mandibule avec le crâne, qui prend place au-dessous du bord antérieur de l'opercule, permet de reconnaître que ces poissons appartiennent à la famille des Myctophidae. La possession d'écailles très fines, le fait que les photophores, qui sont très petits chez cette espèce, ne soient pas observables, conduit à les attribuer à l'espèce Myctophum licatae (Sauvage, 1870), conformément aux conclusions de Gaudant \& Ambroise (1999).

\section{Myctophum (s. 1.) dorsale (Sauvage, 1870)}

Un seul individu très médiocrement conservé, dont la longueur standard égalait environ $35 \mathrm{~mm}$ et dont la hauteur maximale du corps est comprise environ trois fois dans cette dimension, indique la présence de cette espèce dans la diatomite de Molinos de Aguas. Ce fossile, dont il n'est pas possible de donner une description détaillée, diffère de la précédente à la fois par la possession d'écailles cycloïdes épaisses et par celle de photophores relativement gros.

Principaux caractères diagnostiques: conformément aux conclusions de la révision des Myctophidae messiniens de Licata (Gaudant \& Ambroise, 1999), cette espèce est caractérisée par la possession de gros photophores, un caractère qu'elle partage avec l'espèce Myctophum vexillifer (Sauvage, 1870) qui en diffère par sa nageoire dorsale plus grande. Or le spécimen de Molinos de Aguas décrit ci-dessus possède une nageoire anale qui débute en arrière de la verticale passant par la base du dernier rayon de la nageoire dorsale.

Famille Carangidae Rafinesque, 1815

Genre Trachurus Rafinesque, 1810

\section{Trachurus? sp.}

Un seul spécimen juvénile témoigne de la présence d'un Carangidae dans la diatomite de Molinos de Aguas. Sa longueur standard égale $14 \mathrm{~mm}$. Il est caractérisé par sa forme trapue, la hauteur maximale du corps égalant un tiers de la longueur du corps. La tête, massive, constitue plus du tiers de cette dimension.

La colonne vertébrale se compose de 24 vertèbres, dont 14 postabdominales.

La nageoire dorsale est dédoublée. Sa partie épineuse, composée d'épines grêles relativement courtes, débute peu en arrière de la tête. La dorsale postérieure n'est que très imparfaitement fossilisée.

La nageoire anale débute par deux courtes épines en arrière desquelles cinq rayons peuvent seulement être dénombrés. De son endosquelette, on distingue l'axonoste proximal antérieur très long et très redressé qui s'articule avec l'hémapophyse de la première vertèbre postabdominale.
Principaux caractères diagnostiques: bien que cet individu juvénile présente une morphologie très comparable à celle des spécimens de Trachurus trachurus (Linnaeus, 1758) décrits dans d'autres gisements messiniens, notamment à Lorca et en Oranie (Algérie), il est nécessaire de considérer avec réserve la présence de cette espèce dans le Messinien préévaporitique de Molinos de Aguas. En effet, ce spécimen ne présente aucune trace d'écussons le long de la ligne latérale, à la différence des spécimens adultes de l'espèce Trachurus trachurus (Linnaeus, 1758).

Famille Bothidae Regan, 1910

Genre Arnoglossus Bleeker, 1862

\section{Arnoglossus cf. sauvagei d'Erasmo, 1930}

Fig. 4d

Un unique spécimen dépourvu de tête indique la présence d'un Pleuronectiforme à la base de la diatomite de Molinos de Aguas. La longueur du corps sans la tête égale $84 \mathrm{~mm}$, ce qui permet d'estimer à environ $105 \mathrm{~mm}$ la longueur standard. La hauteur maximale du corps égale $30 \mathrm{~mm}$, ce qui indique un corps de forme assez allongée.

La colonne vertébrale comporte au moins 8 vertèbres abdominales dont les centra supportent de longues neurapophyses arquées qui sont toutes légèrement inclinées vers l'arrière, à l'exception de la première qui est faiblement basculée vers l'avant. Trois centra dépourvus de neurapophyses sont également présents plus en avant. Il existe 24 vertèbres postabdominales dont les centra supportent des neurapophyses et des hémapophyses rectilignes relativement longues.

Les dernières vertèbres postabdominales supportent la nageoire caudale de petite taille dont les rayons sont plus ou moins désarticulés.

La nageoire dorsale, dont les premiers rayons étaient insérés au-dessus de la tête, couvrait tout le bord dorsal de l'animal. Ses rayons et son endosquelette sont désarticulés. Il en est de même de la nageoire anale dont les trois axonostes antérieurs sont légèrement déplacés vers l'avant, l'axonoste le plus antérieur ayant perdu son articulation avec l'hémapophyse de la première vertèbre postabdominale. Il est possible d'estimer à au moins une quarantaine le nombre d'axonostes constituant l'endosquelette de la nageoire anale.

Le corps est couvert d'écailles cténoïdes subrectangulaires dont le bord postérieur est orné d'une série de petites épines.

Principaux caractères diagnostiques: son état de conservation médiocre ne permet pas de déterminer avec certitude ce squelette de Pleuronectiforme. Il est toutefois probable qu'on soit en présence d'un représentant de l'espèce $A r$ noglossus sauvagei d'Erasmo, 1930, comme le suggère la composition de sa colonne vertébrale qui comporte 24 vertèbres postabdominales, ce qui exclut qu'il puisse s'agir de l'espèce Microchirus abropteryx (Sauvage, 1870), qui n'en possède que 18. Cependant, selon Landini (1982), l'espèce Arnoglossus sauvagei d'Erasmo, 1930, comporte normalement 27 ou 28 vertèbres postabdominales. C'est pourquoi nous préférons désigner ici le fossile de Molinos de Aguas comme Arnoglossus cf. sauvagei d'Erasmo, 1930. 


\section{SIGNIFICATION PALÉOÉCOLOGIQUE DU MESSINIEN PRÉÉVAPORITIQUE}

Cette ichthyofaune présente une grande similitude avec celle du Cortijo Ruil de Níjar décrite par de la Chapelle \& Gaudant (1987). En effet, dans les deux cas, deux espèces sont fortement dominantes: Myctophum (s. 1.) licatae (Sauvage, 1870) et Maurolicus muelleri (Gmelin, 1789), qui constituent à elles deux 65,3\% de l'ichthyofaune du Cortijo Ruil et qui dominent de manière encore plus nette à Molinos de Aguas (avec 94,6\%), l'espèce Myctophum (s. 1.) licatae (Sauvage, 1870) étant la plus abondante dans les deux gisements. On remarquera en outre que, dans les deux cas, les espèces citées ci-dessus sont représentées par des individus juvéniles dont la longueur standard n'excède généralement pas $35 \mathrm{~mm}$. Ces deux faits conduisent à considérer que les gisements de Molinos de Aguas et du Cortijo Ruil se sont formés dans le même type d'environnement et qu'ils sont vraisemblablement pratiquement contemporains. Cela infirme donc l'hypothèse émise par de la Chapelle \& Gaudant (1987), selon laquelle les diatomites du Cortijo Ruil auraient pu être synchrones de l'épisode évaporitique.
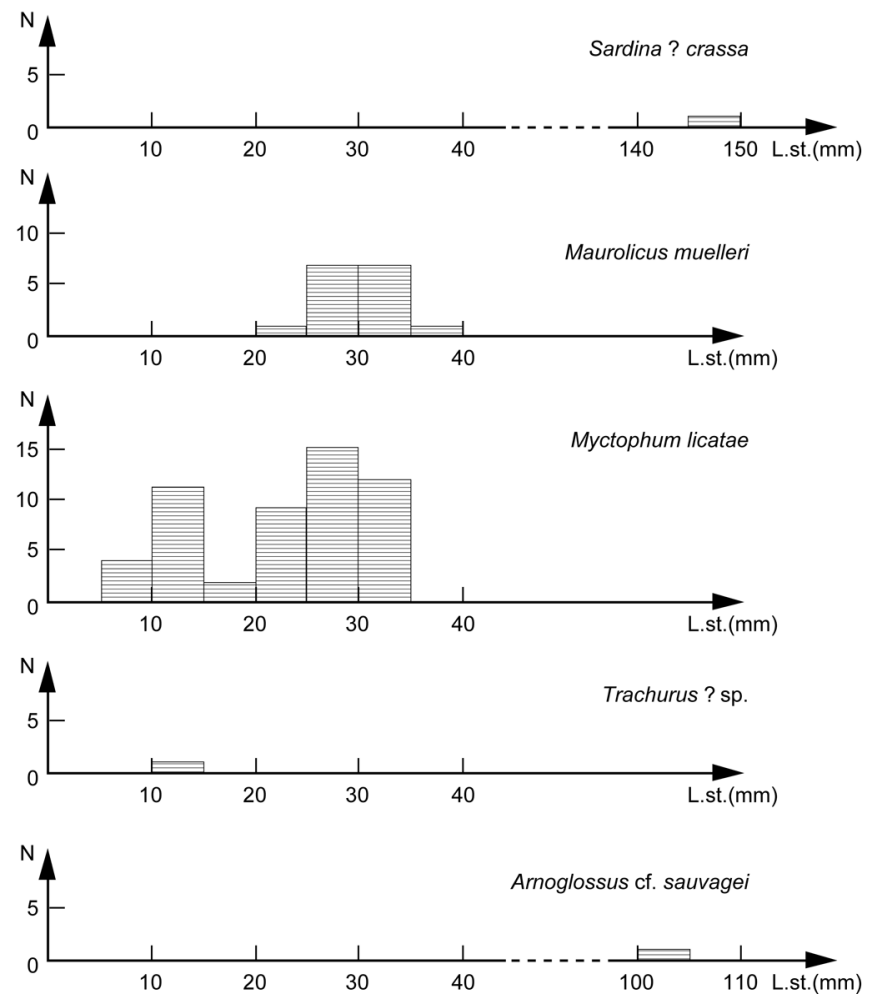

Figure 5. Histogramme des longueurs standard des poissons téléostéens du Messinien préévaporitique ("Formation Abad") de Molinos de Aguas.

Histogramme of standard lengths of the teleostean fishes from the preevaporitic Messinian ("Abad Formation") of Molinos de Aguas.
La principale différence entre les deux gisements tient apparemment au fait que l'ichthyofaune du Messinien préévaporitique de Molinos de Aguas (Fig. 5) est moins diversifiée que celle de Níjar (Fig. 6), ce qui peut s'expliquer par une communication un peu plus restreinte du bassin de Sorbas avec la mer ouverte. La composition de la flore diatomitique de Molinos de Aguas et de Los Yesos, caractérisée par la dominance très marquée de l'espèce Thalassionema nitzschioides Grunow, 1881, qui constitue généralement à elle seule plus de 50\% - et parfois plus de 70\% - du nombre d'individus, indique toutefois sans ambiguité le rôle joué par les upwellings dans la genèse de ces diatomites (SaintMartin et al., 2001). La profondeur relativement faible du dépôt est indiquée par la présence de bivalves dans la marne grise sous-jacente à l'horizon diatomitique fossilifère. Lauriat-Rage (in litt.) y a identifié entre autres Thyasira (s.s.) flexuosa (Montagu, 1803) et Abra (Syndosmya) alba (Wood, 1802), deux espèces qui s'y présentent sous forme, soit de valves en connexion, soit de valves ouvertes à plat
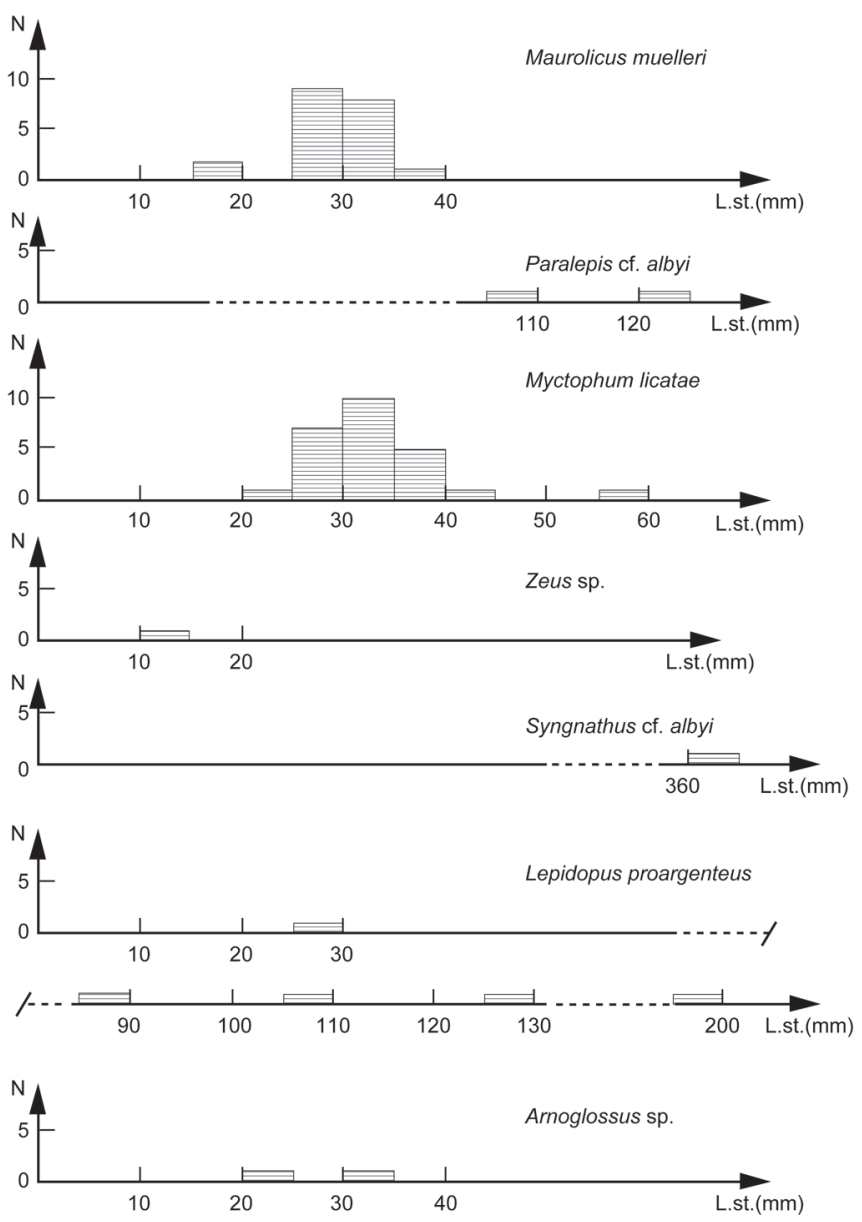

Figure 6. Histogramme des longueurs standard des poissons téléostéens du Messinien préévaporitique du Cortijo Ruil de Níjar.

Histogramme of standard lengths of the teleostean fishes from the preevaporitic Messinian of the Cortijo Ruil of Nijar. 
et non complètement séparées, ce qui indique qu'elles ont été fossilisées sur leur lieu de vie. Ce sont des endobiontes de sédiments fins (vases et sables vaseux) vivant généralement à des profondeurs relativement faibles.

\section{L'ICHTHYOFAUNE DU MESSINIEN INTRAÉVAPORITIQUE}

Plusieurs squelettes plus ou moins fragmentaires de Clupeidae ont été découverts dans un lit d'argilite de cou- leur gris sombre intercalé entre les deux masses de gypse ("Formation Yesares"; Fig. 3) exploitées dans la carrière située à $1 \mathrm{~km}$ au sud-ouest du village de Molinos de Aguas (Néraudeau et al., 2002).

Le seul spécimen pratiquement entier porte le numéro de catalogue MNHNP-PTE 470A (Fig. 7a). C'est un jeune individu dont la longueur standard égale $41 \mathrm{~mm}$. Il est caractérisé par la forme anormalement effilée de son corps dont la hauteur maximale égale seulement 1/8 de la longueur standard. En revanche, le corps d'un second individu (MNHNP-PTE 471), dont seule la moitié antérieure du corps est conservée et dont la longueur standard mesurait environ $40 \mathrm{~mm}$, possédait des proportions plus
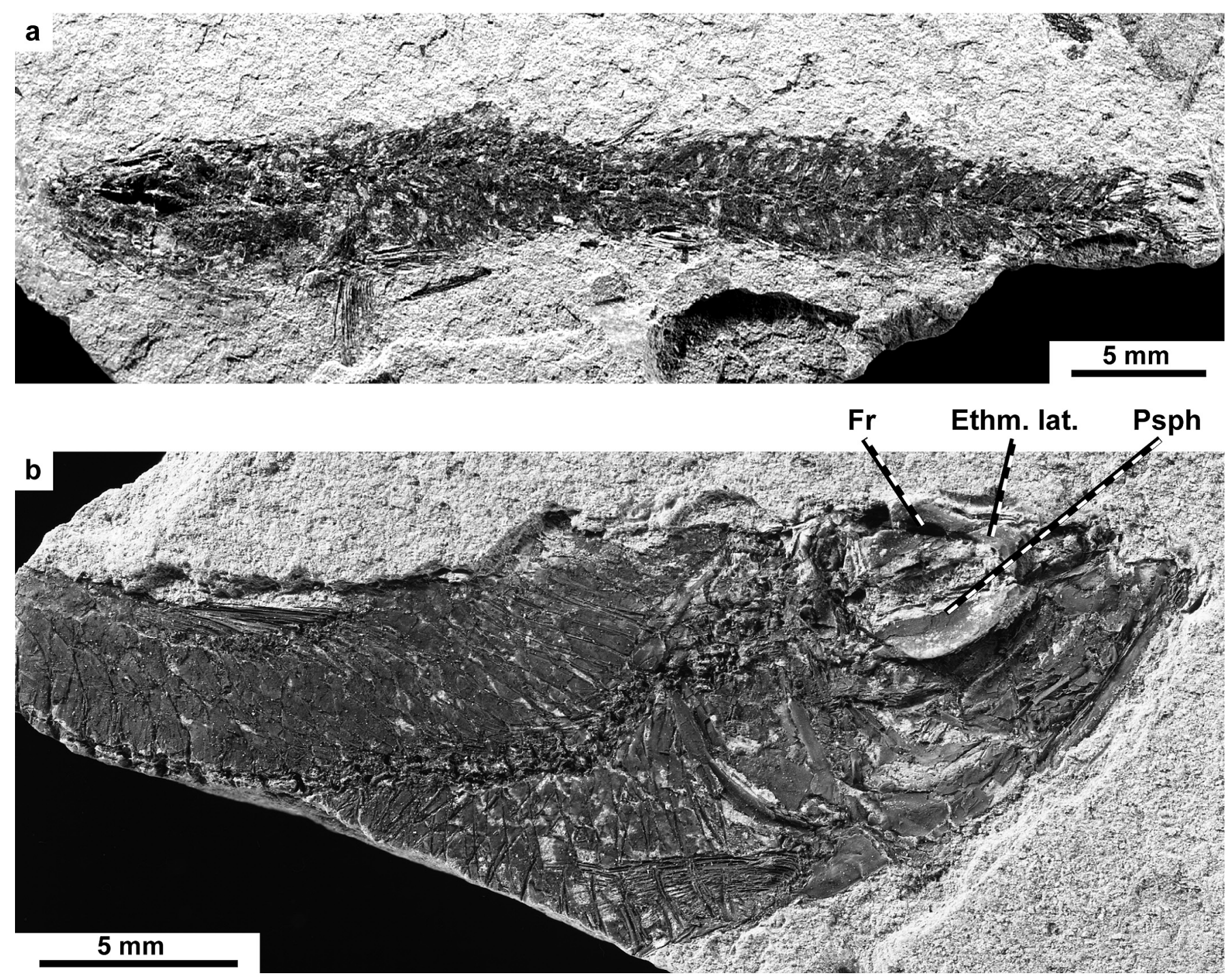

Figure 7. Sardina ? crassa (Sauvage, 1873). Messinien intraévaporitique ("Formation Yesares") de la carrière de gypse de Molinos de Aguas (route de Hueli). a, Vue générale du spécimen MNHNP-PTE 470, conservé à Paris dans les collections paléontologiques du Muséum national d'Histoire naturelle. b, Spécimen MNHNP-PTE 471, conservé à Paris, dans les collections du laboratoire de Paléontologie du Muséum national d'Histoire naturelle. Des traces de pachyostose sont visibles sur le neurocrâne. Ethm. lat.: ethmoïde latéral; Fr: frontal; Psph: parasphénoïde [Clichés D. Serrette].

Sardina ? crassa (Sauvage, 1873). Intraevaporitic Messinian ("Yesares Formation") of the gypsum quarry of Molinos de Aguas (road to Hueli). a, General view of specimen MNHNP-PTE 470, kept in Paris, in the palaeontological collections of the National Museum of Natural History. b, Specimen MNHNP-PTE 471, kept in Paris, in the palaeontological collections of the National Museum of Natural History. The occurrence of pachyostosis is visible in the neurocranium [Cliché D. Serrette]. 
conformes à la norme car la hauteur maximale de son corps devait être comprise environ quatre fois dans la longueur standard. Sa tête (Fig. 7b), relativement massive, puisque sa hauteur excède les $3 / 4$ de sa longueur, montre des traces de pachyostose observables sur le parasphénoïde - comme c'est également le cas sur le spécimen MNHNP-PTE 470 -, mais également sur l'ethmoïde latéral et l'orbitosphénoïde. L'opercule, subrectangulaire, observable sur le spécimen MNHNP-PTE 472, est orné sur son champ antéro-ventral de rides rayonnantes.

La colonne vertébrale est composée d'environ 45 vertèbres, sans qu'il soit possible de préciser le nombre de vertèbres postabdominales. La nageoire dorsale, dont la composition est inconnue, débute au milieu de la longueur du corps, mesurée de la pointe du museau au bord postérieur des hypuraux. Les nageoires pectorales qui comportent une quinzaine de rayons, occupent une position basse sur le flanc. Les nageoires pelviennes sont insérées un peu en arrière de la verticale passant par l'origine de la dorsale. La nageoire pectorale, qui est composée d'une quinzaine de rayons, occupe une position basse sur le flanc. Le spécimen MNHNPPTE 472 possède une carène ventrale comportant une douzaine d'écussons entre les nageoires pectorales et pelviennes.

Principaux caractères diagnostiques: Malgré ses lacunes, la description précédente a permis d'établir que les poissons découverts dans un lit d'argilite euxinique de la carrière de gypse de Molinos de Aguas sont des Clupeidae appartenant à l'espèce Sardina ? crassa (Sauvage, 1873), comme l'indiquent notamment la possession d'un opercule orné de stries rayonnantes, d'une colonne vertébrale composée d'environ 45 vertèbres et d'une carène ventrale protégeant le bord ventral de la cavité abdominale.

\section{Signification paléoécologique}

Cette espèce marine est présente dans un certain nombre de gisements du Messinien préévaporitique: elle a notamment été signalée au Maroc, aux environs de MelillaNador (gisements de Rostro Gordo et d'El Koulla) (Gaudant et al., 1994b), à Oran (gisements de Raz el Aïn et de Gambetta-Ravin blanc) (Arambourg, 1927; Gaudant, 2008), à Lorca, Campos del Río et Hurchillo (Espagne) (Gaudant, 1995; Gaudant et al., 1994a; Gaudant et al., 2001). Elle a également été observée dans les gypses lités (gypsarénites) de Crète occidentale où elle est associée à de très nombreux représentants de l'espèce Aphanius crassicaudus (Agassiz, 1839) (Gaudant, 1980).

Un rapprochement peut également être établi avec les Sardina ? crassa (Sauvage, 1873) fossilisés dans une diatomite messinienne du Djebel Murdjadjo (Oranie, Algérie) qui, comme les spécimens récoltés dans la carrière de gypse de Molinos de Aguas, possèdent des os céphaliques affectés de pachyostose (Gaudant \& Meunier, 1996; Gaudant et al., 1997). Or, cette diatomite appartient précisément à une unité sédimentaire qui renferme des faciès évaporitiques dans la région d'Oran (Mansour \& SaintMartin, 1999). Bien qu'elle soit la seule espèce de poissons présente dans le banc d'argilite grise intercalée entre les deux masses de gypse sélénite exploitées dans la car- rière de Molinos de Aguas, Sardina ? crassa y témoigne de la persistance, dans les environs, d'une mer à salinité normale pendant le dépôt de ces gypses.

On rappellera ici que la présence de fossiles marins dans des horizons marneux ou calcaires intercalés entre les bancs de gypse messinien, est connue depuis plus d'un quart de siècle dans le bassin de Sorbas (Province d'Almería, Espagne), notamment grâce aux travaux de Schleich (1977), de Montenat et al. (1980) et d'Ott d'Estevou (1980) (cf. Ott d'Estevou \& Montenat, 1990). Deux sites en particulier ont été étudiés de manière approfondie: Los Yesos, à une quinzaine de kilomètres à l'ouest-sud-ouest de Sorbas, qui a fait récemment l'objet de nouvelles recherches (Saint-Martin et al., 2000), et Molinos de Aguas, à environ 4 kilomètres au sud-sud-est de Sorbas (Néraudeau et al., 2002). On soulignera toutefois qu'aucun reste squelettique de poissons n'a encore été découvert dans les interlits des gypses messiniens de Los Yesos.

Néanmoins, la découverte de Sardina ? crassa (Sauvage, 1973) dans le Messinien intraévaporitique de Molinos de Aguas vient renforcer, s'il en était besoin, les conclusions de Montenat et al. (1980) pour qui "la présence de microfaunes et de macrofaunes non remaniées dans les sédiments alternant avec les gypses atteste la possibilité de liaisons directes avec le milieu marin pendant l'épisode évaporitique", conclusions confirmées par Saint-Martin et al. (2000) qui soulignent que "l'hypothèse d'une dessiccation complète de la Méditerranée avant la formation des gypses dans le bassin de Sorbas n'apparaît pas compatible avec une certaine continuité des peuplements benthiques". Plus généralement, cette découverte s'accorde parfaitement avec les observations réalisées précédemment, à l'échelle de la Méditerranée occidentale, sur la diatomite messinienne de Castagnito (Piémont), intercalée entre deux bancs de gypse (Fourtanier et al., 1991) et celles relatives à l'ichthyofaune messinienne décrite il y a quelques années dans une diatomite du djebel Murdjadjo, près d'Oran (Algérie) (Gaudant et al., 1997), que Mansour \& SaintMartin (1999) considèrent comme synchrones de "la base de sédiments renfermant des faciès évaporitiques dans la région d'Oran".

Notons cependant que le caractère monospécifique de l'ichthyofaune et l'existence d'un phénomène de pachyostose affectant certains os céphaliques des poissons du Messinien intraévaporitique de Molinos de Aguas suffisent à démontrer que ceux-ci ne vivaient pas dans une eau marine normale.

\section{L'ICHTHYOFAUNE DU MESSINIEN POSTÉVAPORITIQUE}

La découverte par Gilles Conesa, entre Molinos de Aguas et Sorbas, dans les laminites marno-silteuses qui surmontent la formation gypseuse, d'un individu dont la 
longueur standard égale $43 \mathrm{~mm}$ (Fig. 8), témoigne de la présence de l'espèce Aphanius crassicaudus (Agassiz, 1839) dans le Messinien postévaporitique ("Formation Sorbas"; Fig. 3). Cette découverte n'est pas pour surprendre car elle confirme les observations faites précédemment par Gaudant \& Ott d'Estevou (1985) dans des faciès similaires, à environ 2 kilomètres plus au nord.

Nous mettons à profit cette opportunité pour signaler ici qu'en étudiant le Messinien aux environs du village de Gafares, situé à environ $8,5 \mathrm{~km}$ à l'ouest de Carboneras (Fig. 1), dans le bassin voisin de Níjar-Carboneras, JeanMarie Rouchy a observé la présence de squelettes articulés de poissons téléostéens dont un rapide examen nous a permis de constater qu'il s'agit également de représentants de la famille des Cyprinodontidae appartenant à l'espèce Aphanius crassicaudus (Agassiz, 1839). Cette espèce avait été précédemment signalée il y a une vingtaine d'années à environ un kilomètre au sud-ouest de Gafares, dans la Rambla de Las Colmenas (de la Chapelle \& Gaudant, 1987). Selon Jean-Marie Rouchy, la coupe exposée à quelques centaines de mètres à l'est du hameau de Gafares, dans la Loma de Los Yesares, permet d'observer une succession sédimentaire continue qui couvre la majeure partie du Messinien. Ces dépôts ont été décrits du point de vue lithologique, sédimentologique et micropaléontologique par Van de Poel (1991), Fortuin \& Krijgsman (2003) et Bassetti et al. (2006). La coupe, qui correspond à celle décrite par Fortuin \& Krijgsman (2003: fig. 2 et 5, cf. section E) débute par les alternances de marnes et de diatomites classiquement rapportées à la formation du tripoli ("Formation Abad"), suivie, sur une centaine de mètres d'épaisseur, par une alternance de huit bancs de gypse d'épaisseur plurimétrique et de couches de sédiments silteux. Cet ensemble passe verticalement à une série de silts, de sables, de conglomérats et de niveaux de calcai- re dolomitique vacuolaire dont les vides correspondent à des moules de cristaux de gypse. La série se termine par des dépôts très riches en oxyde de manganèse. Dans la nomenclature lithostratigraphique régionale, cet ensemble correspond à la "Formation Yesares" et, pour Van de Poel (1991) à une sous-unité appelée "Manco Limestone", qui est propre au bassin de Nijar-Carboneras où elle est recouverte par la "Formation Feos" qui est considérée comme l'équivalent de l'intervalle dit du "lago-mare" des autres bassins méditerranéens et notamment à la "Formation Sorbas" du bassin voisin. C'est dans la partie terminale de la "Formation Yesares", et plus précisément dans 1'“Upper Manco limestone" (Bassetti et al., 2006), que des squelettes de poissons ont été découverts lors d'une mission de terrain réalisée en 1998 (Fig. 9). Le niveau fossilifère est localisé à environ un mètre au-dessous du dernier banc de carbonates vacuolaires à moules de cristaux de gypse et trois mètres sous la base des sédiments noirs riches en manganèse. Si l'interprétation stratigraphique proposée par Van de Poel (1991), Fortuin \& Krijgsman (2003) et Bassetti et al. (2006) est correcte, ce gisement serait donc un peu plus ancien que les niveaux à Aphanius crassicaudus (Agassiz) du bassin de Sorbas, qui se situent dans la partie inférieure de la "Formation Sorbas".

Nous avons récolté une quarantaine de spécimens dans ce gisement que nous nommons ici "Gafares Pueblo". Leur longueur standard varie de 17 à $56,5 \mathrm{~mm}$ avec une fréquence maximale entre 35 et $40 \mathrm{~mm}$, comme c'est le cas dans la Rambla de Las Colmenas (Fig. 10). Le corps est allongé : sa hauteur est généralement comprise 4 ou 5 fois dans la longueur standard. La tête, massive, constitue entre $1 / 4$ et $1 / 3$ de la longueur standard. Des dents tricuspides sont observables sur le bord oral du prémaxillaire.

La colonne vertébrale dont la composition n'a pas pu être précisée, comporte généralement 15 ou 16 vertèbres postabdominales. Les côtes pleurales sont au nombre de 8 ou 9 paires.

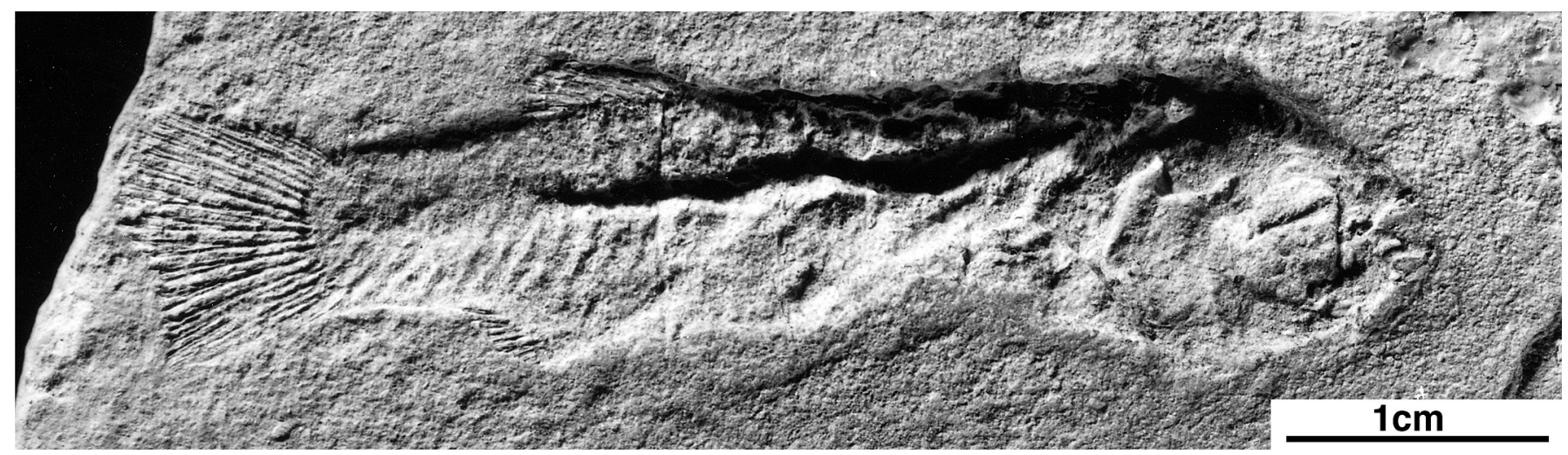

Figure 8. Aphanius crassicaudus (Agassiz, 1839). Spécimen récolté dans le Messinien postévaporitique ("Formation Sorbas") entre Molinos de Aguas et Sorbas. Spécimen MNHNP-PTE 478, conservé à Paris dans les collections paléontologiques du Muséum national d'Histoire naturelle [Cliché D. Serrette].

Aphanius crassicaudus (Agassiz, 1839). Specimen collected in the post-evaporitic Messinian ("Sorbas Formation") between Molinos de Aguas and Sorbas. Specimen MNHNP-PTE 478, kept in Paris, in the palaeontological collections of the National Museum of Natural History [Cliché D. Serrette]. 
Sur tous les individus, les vertèbres et les côtes pleurales sont plus ou moins fortement affectées par la pachyostose, comme cela est le cas chez les Aphanius crassicaudus (Agassiz, 1839) de Las Lomas de Sorbas (Gaudant \& Ott d'Estevou, 1985; Meunier \& Gaudant, 1987) et de la Rambla de Las Colmenas (de la Chapelle \& Gaudant, 1987).

La nageoire dorsale, qui est généralement composée de 9 ou 10 rayons, dont 7 ou 8 sont à la fois articulés et bifurqués, est soutenue par un endosquelette composé d'environ 8 axonostes proximaux. Elle est toujours insérée sensiblement en avant de la verticale passant par l'origine de l'anale. La distance antédorsale égale généralement 62 à $66 \%$ de la longueur standard, contre 66 à $73 \%$ pour la distance antéanale. La nageoire anale qui comporte 9 ou 10 rayons, dont 7 ou 8 sont à la fois articulés et bifurqués, est soutenue par un endosquelette formé d'environ 8 axonostes proximaux. La nageoire caudale, en forme de palette faiblement arrondie à son extrémité distale, comporte environ 14 rayons principaux à la fois articulés et bifurqués.

Le corps est couvert d'écailles cycloïdes dont la surface est ornée de circuli concentriques.
Principaux caractères diagnostiques: la présence de dents tricuspides sur les mâchoires, la composition de la colonne vertébrale, la position relative et la composition des nageoires dorsale et anale permettent d'affirmer que le matériel recueilli près de Gafares appartient bien à l'espèce Aphanius crassicaudus (Agassiz, 1839), comme c'est le cas pour le spécimen recueilli par Gilles Conesa entre Sorbas et Molinos de Aguas et également pour le matériel de Las Lomas de Sorbas étudié par Gaudant \& Ott d'Estevou, 1985)

\section{CONCLUSION}

Le bassin de Sorbas diffère de tous les autres bassins messiniens du sud-est de l'Espagne ayant livré une ichthyofaune par le fait qu'il renferme trois niveaux fossilifères superposés ayant livré des squelettes de poissons.
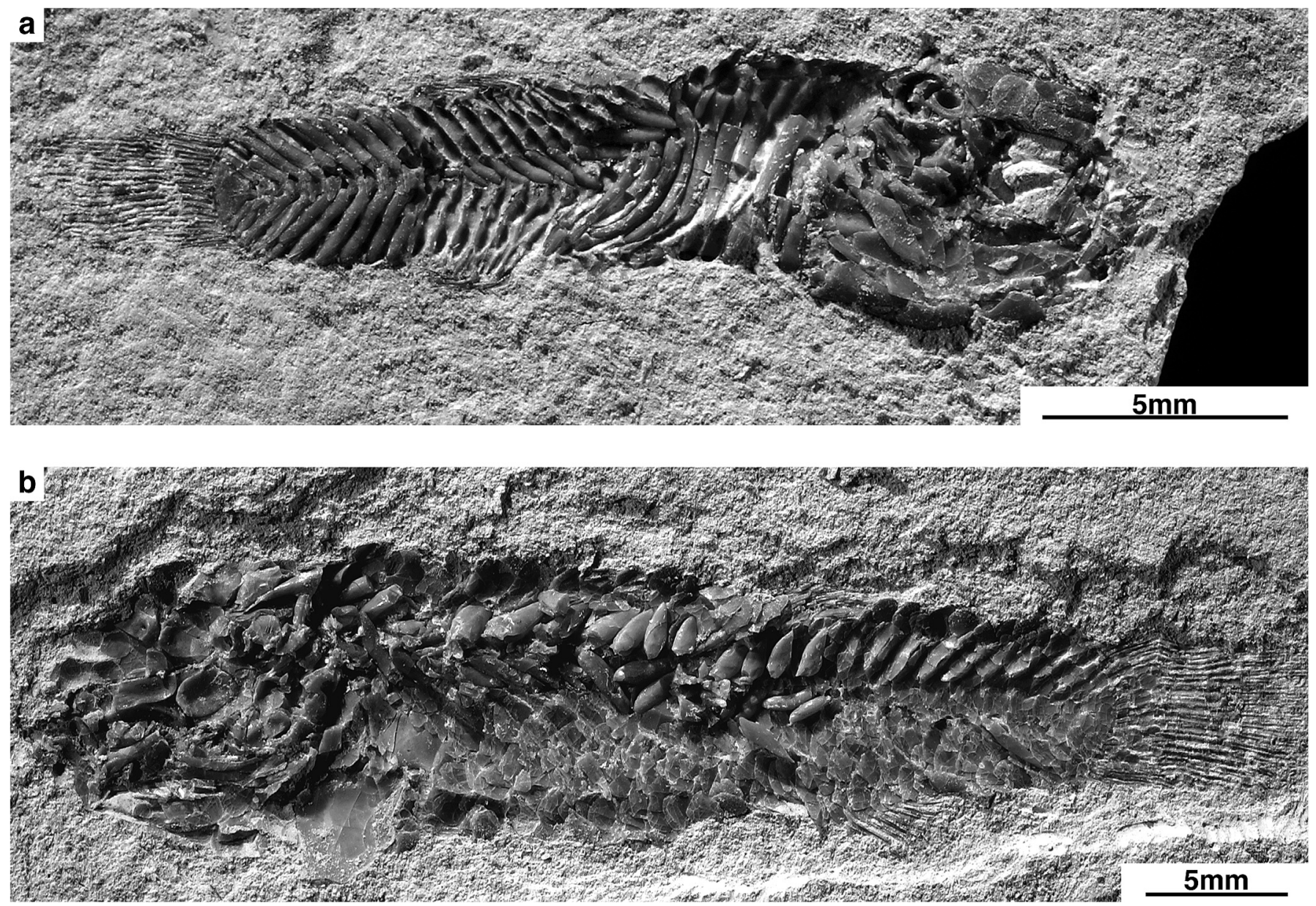

Figure 9. Aphanius crassicaudus (Agassiz, 1839). Deux spécimens provenant du "Upper Manco Limestone" de la Loma de Los Yesares ("Gafares Pueblo"). Spécimens MNHNP-NIJ 117A (en haut) and NIJ 114A (en bas), conservés à Paris, dans les collections paléontologiques du Muséum national d'Histoire naturelle [Cliché D. Serrette].

Aphanius crassicaudus (Agassiz, 1839). Two specimens from the "Upper Manco limestone" of the Loma de Los Yesares ("Gafares Pueblo"). Specimens MNHNP-NIJ 117A (above) and NJJ 114A (below), kept in Paris, in the palaeontological collections of the National Museum of Natural History [Cliché D. Serrette]. 


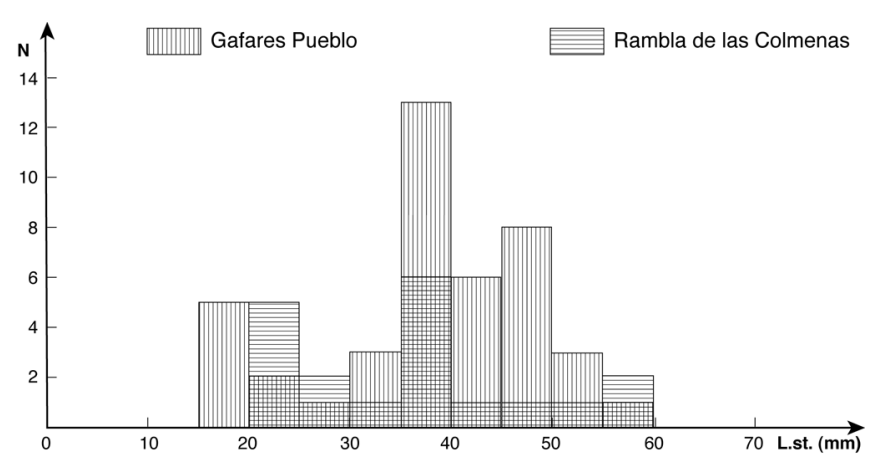

Figure 10.Histogramme des longueurs standard des Aphanius crassicaudus (Agassiz, 1839) récoltés dans les gisements de la Rambla de las Colmenas et de la Loma de los Yesares ("Gafares Pueblo").

Histogramme of standard lengths of Aphanius crassicaudus (Agassiz, 1839) collected at Rambla de las Colmenas and at Loma de los Yesares ("Gafares Pueblo").

Il fournit ainsi des éléments permettant de reconstituer l'histoire de cette région pendant la crise fini-miocène de la Paléoméditerranée :

$1^{\circ}$ Les diatomites préévaporitiques de Molinos de Aguas et de Los Yesos ("Formation Abad") qui constituent l'équivalent latéral de celles du Cortijo Ruil de Níjar (de la Chapelle \& Gaudant, 1987) traduisent l'existence à faible distance d'une mer profonde peuplée de nombreux Myctophidae et affectée de phénomènes d'upwellings.

$2^{\circ}$ L'argilite intra-évaporitique de la carrière de gypse de Molinos de Aguas ("Formation Yesares"), qui a livré uniquement l'espèce Sardina ? crassa (Sauvage, 1873), témoigne de la persistance d'un milieu marin pendant le dépôt des gypses.

$3^{\circ}$ Les laminites marno-silteuses superposées aux gypses ("Formation Sorbas") renferment l'espèce Aphanius crassicaudus (Agassiz, 1839), caractéristique des faciès lagunaires du Messinien. Leur présence indique donc qu'un abaissement significatif du niveau marin s'est produit après le dépôt des gypses dans les intercalations sableuses desquels sont fossilisés de nombreux foraminifères et des restes d'invertébrés marins: bryozoaires, bivalves, gastéropodes, serpules, échinides, crustacés et ostracodes (Saint-Martin et al., 2000).

\section{REMERCIEMENTS}

L'intérêt de l'auteur pour le site de Molinos de Aguas a été suscité il y a une vingtaine d'années par Philippe Ott d'Estevou qui lui a indiqué cet affleurement à une époque où la végétation qui le couvrait empêchait d'y réaliser des fouilles.

L'auteur remercie Gilles Conesa qui lui a communiqué le spécimen d'Aphanius crassicaudus qu'il a découvert dans le Messinien postévaporitique du même secteur. Cette espèce est également présente aux environs de Gafares, dans le bassin de Níjar-Carboneras où Jean-Marie Rouchy a bien voulu conduire l'auteur sur un nouvel affleurement fossilifère et lui communiquer la description de la coupe du gisement de Gafares Pueblo qui est reproduite ci-dessus.

Enfin, l'auteur est reconnaissant envers Agnès Lauriat-Rage qui a accepté d'étudier les bivalves du Messinien préévaporitique de Molinos de Aguas.

L'illustration a été préparée par M. Joël Dyon.

\section{BIBLIOGRAPHIE}

Arambourg, C. 1927. Les poissons fossiles d'Oran. Matériaux pour la Carte géologique d'Algérie, $1^{\mathrm{e}}$ série, Paléontologie, 6, 1-298, atlas.

Bassetti, M.A., Miculan, P. \& Sierro, F.J. 2006. Evolution and depositional environments after the end of Messinian Salinity Crisis in Níjar basin (SE Betic Cordillera). Sedimentary Geology, 188-189, 279-295.

Chapelle, G. de la \& Gaudant, J. 1987. Découverte de deux nouveaux gisements de poissons fossiles messiniens dans le bassin de Níjar-Carboneras (Andalousie orientale): signification paléoécologique et implications paléogéographiques. Estudios geológicos, 43, 279-297.

Dronkert, H. 1978. Late Miocene evaporites in the Sorbas basin and adjoining areas. Memorie della Società italiana di Geologia, 16, year 1976, 341-361.

Fortuin, A.R. \& Krijgsman, W. 2003. The Messinian of the Níjar Basin (SE Spain): sedimentation, depositional environments and paleogeographic evolution. Sedimentary Geology, 160, 213-242.

Fourtanier, E., Gaudant, J. \& Cavallo, O. 1991. La diatomite de Castagnito (Piémont): une nouvelle preuve de l'existence d'oscillations modérées du niveau marin pendant le Messinien évaporitique. Bollettino della Società Paleontologica Italiana, 30, 79-95.

Gaudant, J. 1980. Sur la présence d'Alosa crassa Sauvage (Poissons téléostéens, Clupeidae) dans les gypses messiniens de Crète occidentale. Proceedings of the koninkijke Nederlandse Akademie van Wetenschappen, (B), 83, 263-268.

Gaudant, J. 1995. Nouvelles recherches sur l'ichthyofaune messinienne de Lorca (Murcia, Espagne). Revista Española de Paleontología, 10, 175-189 [Corrección, 1996 Ibid., 11, 122-123].

Gaudant, J. 2008. Paléobiodiversité et paléoenvironnements: l'exemple des gisements de poissons téléostéens du Messinien préévaporitique d'Oran et du bassin du Chélif (Algérie). Geodiversitas, 30, 141-163.

Gaudant, J. \& Ambroise, D. 1999. Réexamen critique des Myctophidae (Poissons téléostéens) messiniens de Licata (Sicile, Italie): conséquences taxonomiques. Cybium, 23, 131-145.

Gaudant, J., Courme, M.-D. \& Marín Ferrer, J. M. 2001. Hurchillo (Province d'Alicante, Espagne): un gisement de poissons messiniens d'un type encore inconnu. Comptes Rendus de l'Académie des Sciences, Paris, 333 (IIa), 411-417. 
Gaudant, J., Loiseau, J. \& Ott d'Estevou, Ph. 1994a. Découverte d'une frayère fossile de poissons téléostéens dans le Messinien des environs de Campos del Río (Province de Murcia, Espagne). Revista Española de Paleontología, 9, 37-50.

Gaudant, J. \& Meunier, F. 1996. Observation d'un cas de pachyostose chez un Clupeidae fossile du Miocène terminal de l'ouest algérien, Sardina ? crassa (Sauvage, 1873). Cybium, 20, 169-183.

Gaudant, J. \& Ott d'Estevou, Ph. 1985. Première découverte d'Aphanius crassicaudus (Agassiz) (Poisson téléostéen, Cyprinodontidae) dans le Messinien post-évaporitique d'Andalousie. Estudios geológicos, 41, 93-98.

Gaudant, J., Saint-Martin, J.-P., Benmoussa, A., Cornée, J.-J., El Hajjaji, K. \& Muller, J. 1994b. L'ichthyofaune messinienne à la périphérie de la plateforme carbonatée de Melilla-Nador (Nord-Est du Maroc). Géologie méditerranéenne, 21, 25-35.

Gaudant, J., Saint-Martin, J.-P., Bessedik, M., Mansour, B., Moissette, P. \& Rouchy, J.-M. 1997. Découverte d'une frayère de poissons téléostéens dans des diatomites messiniennes du Djebel Murdjadjo (environs d'Oran, Algérie). Journal of African Earth Sciences, 24, 511-529.

Landini, W. 1982. I Pleuronectiformi (Pisces Teleostea) fossili del Neogene italiano. Atti della Società Toscana di Scienze naturali, Memorie, (A), 88, 1-41.

Landini, W. \& Menesini, E. 1980. Studi sulle ittiofaune messiniane. II. Studio sistematico di Maurolicus muelleri (Gmelin) (fam. Gonostomatidae). Atti della Società Toscana di Scienze naturali, Memorie, (A), 87, 231-255.

Mansour, B. \& Saint-Martin, J.-P. 1999. Conditions de dépôt des diatomites messiniennes en contexte de plate-forme carbonatée d'après l'étude des assemblages de diatomées: exemple du Djebel Murdjadjo (Algérie). Geobios, 32, 395-408.

Meunier F.J. \& Gaudant J. 1987. Sur un cas de pachyostose chez un poisson du Miocène terminal du bassin méditerranéen, Aphanius crassicaudus (Agassiz), (Teleostei, Cyprinodontidae). Comptes Rendus de l'Académie des Sciences, Paris, 305 (II), 925-928.

Montenat, C., Ott d'Estevou, Ph., Plaziat, J.-C. \& Chapel J. 1980. La signification des faunes marines contemporaines des évaporites messiniennes dans le Sud-Est de l'Espa- gne. Conséquences pour l'interprétation des conditions d'isolement de la Méditerranée occidentale. Géologie méditerranéenne, 7, 81-90.

Néraudeau, D., Videt, B., Courville, Ph., Goubert, E. \& Rouchy, J.-M. 2002. Corrélation des niveaux fossilifères marins interstratifiés dans les gypses messiniens, entre la carrière de Los Yesos et la carrière de Molinos de Aguas (bassin de Sorbas, SE Espagne). Geodiversitas, 24, 659-667.

Ott d'Estevou, Ph. 1980. Evolution dynamique du bassin néogène de Sorbas (Cordillères bétiques orientales, Espagne). Documents et Travaux de l'Institut géologique Albert de Lapparent, 1, 1-264.

Ott d'Estevou, Ph. \& Montenat, C. 1990. Le bassin de Sorbas - Tabernas. In: Les bassins néogènes du domaine bétique oriental (Espagne) (coord. C. Montemat). Documents et Travaux de l'Institut géologique Albert de Lapparent, 12-13, 101-128.

Saint-Martin, J.-P., Néraudeau, D., Lauriat-Rage, A., Goubert, E., Secrétan, S., Babinot, J.-F., Boukli-Hacène, S., Pouyet, S., Lacour, D., Pestrea, S. \& Conesa, G. 2000. La faune interstratifiée dans les gypses messiniens de Los Yesos (bassin de Sorbas, SE Espagne): implications. Geobios, 33, 637-649.

Saint-Martin, J.-P., Pestrea, S. \& Conesa, G. 2001. Les assemblages de diatomées des niveaux infra-gypseux du bassin messinien de Sorbas (Espagne). Cryptogamie, Algologie, 22, 127-149.

Schleich, H.H. 1977.Geologic and paleontologic investigations of the Messinian gypsum deposits and their surrounding sediments at Tabernas (Almeria, SE Spain). Messinian seminar N. 3, Malaga, Guidebook, 64-68.

Sierro, F.J., Hilgen, F.J., Krijgsman, W. \& Flores, J.A. 2001. The Abad composite (SE Spain): a Messinian reference section for the Mediterranean and the APTS. Palaeogeography, Palaeoclimatology, Palaeoecology, 168, 141-169.

Van de Poel, H.M. 1991. Messinian stratigraphy of the Níjar Basin (S.E. Spain) and the origin of its gypsum-ghost limestones. Geologie en Mijnbouw, 70, 215-234.

Manuscrito recibido: 2 de Agosto, 2007 Manuscrito aceptado: 4 de Septiembre, 2008 
